\title{
Gout involved the cervical disc and adjacent vertebral endplates misdiagnosed infectious spondylodiscitis on imaging: case report and literature review
}

\author{
Suying Zhou, Yundan Xiao, Xin Liu, Yi Zhong and Haitao Yang ${ }^{*}$ (D)
}

\begin{abstract}
Background: Gout in spine is rare and commonly mimics some infectious or tumoral lesions, the differentiation of spinal gout from other diseases is not always easy. We report a case of gout involved cervical disc and adjacent vertebral endplates whose etiology was initially not determined. Compared with the previous published 10 similar cases, this case displayed a complete and continuous image data with higher image quality and resolution than before. So we give a brief literature review for concerning cervical gout, with the emphasis on the discussion of radiological findings.

Case presentation: A 50-year-old male with a 5-year history of neck and shoulder pain had muscle atrophy and weakness in both arms. Physical examination revealed multiple tophi were seen in left wrist, both feet and knee; bilateral superficial sensory declined below level of mastoid portion and the muscle strengths of limbs decreased. Laboratory findings showed hyperuricemia and the C-reactive protein level was very high. Imaging studies including Computed Tomography (CT) and Magnetic Resonance Imaging (MRI) showed abnormality of the C5-6 intervertebral disc and irregular osteolytic destruction of both adjacent C5-6 endplates, narrowing of C5-6 disc space and swelling of prevertebral soft tissue. Under the circumstance of the lesions being not determined and nerve root symptoms, surgical treatment was performed and pathological examination of the specimen revealed deposited uric acid crystals surrounded by granulomatous inflammation. After surgery combined with pharmaceutical and rehabilitation treatment, the muscle strengths of limbs, the pain of neck and shoulder and the level of serum uric acid were all improved.

Conclusions: Cervical spinal gout involving the disc and adjacent vertebral endplates is uncommon and may misunderstand infectious spondylodiscitis. Physician and radiologist should take the gouty spondylitis into account with a combination with previous history and clinical manifestations when encountering with such this condition.
\end{abstract}

Keywords: Spine, Cervical, Gout, Radiology

\section{Background}

Gout is a metabolic disorder characterized by hyperuricemia and abnormal depositions of urate around a variety of tissues, more frequently including peripheral joints of the upper and lower extremities, typically in the first metatarsophalangeal joint [1]. Gouty involvement in the axial skeleton is a seemingly uncommon manifestation.

Any segment of the spine and its components (vertebral bodies, pedicles, lamina, ligaments, interapophyseal

\footnotetext{
* Correspondence: frankyang119@126.com

Department of Radiology, the First Affiliated Hospital of Chongqing Medical University, 1 Youyi Road, Yuzhong District, Chongqing 400016, China
}

cartilage, and epidural and intradural spaces) may be involved, with lumbar involvement being the most common $[2,3]$. It has been reported by Michael et al. that most cases $(80.6 \%)$ only involved one region of the spine and $24.8 \%$ affected only the cervical spine [1]. Gout involving the endplates of two contiguous vertebral bodies and intervertebral disc is rare, which frequently mimics some degenerative changes and infectious spondylodiscitis on imaging, leading to delayed diagnosis and treatments. Therefore, the main imaging differentiation factors between these conditions should be further investigated. Up to now, there have only been 10 cervical

(c) The Author(s). 2019 Open Access This article is distributed under the terms of the Creative Commons Attribution 4.0 International License (http://creativecommons.org/licenses/by/4.0/), which permits unrestricted use, distribution, and reproduction in any medium, provided you give appropriate credit to the original author(s) and the source, provide a link to the Creative Commons license, and indicate if changes were made. The Creative Commons Public Domain Dedication waiver (http://creativecommons.org/publicdomain/zero/1.0/) applies to the data made available in this article, unless otherwise stated. 
gout cases with images reported in the English-language literature searched in PubMed [3-11]; most of them showed the incomplete image sequence and poor image quality which were insufficient to understand the imaging manifestations of this condition for improving diagnostic accuracy. In this article, we report a case about a spinal gout affected the cervical disc and adjacent endplates with a complete and continuous image data with higher image quality and resolution than previous published cases, whose etiology was initially not completely determined and suspected as infectious spondylodiscitis, and provide a brief literature review concerning cervical gout. In addition, the case report is prepared and reported in accordance with CARE-checklist [12].

\section{Case presentation}

\section{Patient information}

A 50-year-old Chinese man with a 5-year history of neck and shoulder pain presented with muscle atrophy and weakness in both arms. He was admitted to hospital with complaints of unstable holding for 9 months and numbness of limbs for 2 months, which worsened in one week. Five years previously, he tried the physiotherapy due to the pain of neck and shoulder, and relief from pain followed. Nine months previously, muscle atrophy and weakness in both arms were noted but without being promptly treated. And his pain of neck was obviously worsened 2 months before admission, together with numbness of limbs; the cervical and lumbar image examinations in other hospital were suggestive of protrusion of intervertebral disc at the C5-7 level and the L4-S1 level. Then he received the physiotherapy and felt the relief of pain, but numbness of limbs did not significantly relieve, which suddenly worsened in one week.

The patient also presented with a 3-year history of diabetes mellitus (DM), a 20-year history of gout involving left wrist and left knee, a 30-year history of smoking and drinking. Previous pharmaceutical treatment for gout had been intermittent for about 1 year, consisting of dexamethasone for acute episodes. Patient's family and psychosocial history were not relevant for this case report.

\section{Clinical findings}

After admission, physical examination was performed in accordance with best practice and clinical guidelines [13] by physician, in which multiple tophi were seen in left wrist, both feet and knee; bilateral superficial sensory declined below the level of mastoid portion, particularly below the knee, which presumably resulted from the lumbar lesions. The muscle strengths of upper limbs were graded $2+$ to 4 and lower limbs were graded 0 in accordance with Medical Research Council (MRC) Scale for Muscle Strength [14]. The reflex of bilateral biceps, triceps and radial periosteum disappeared; the reflex of bilateral knee was normal and that of bilateral ankle disappeared. The muscle atrophy of bilateral thenar, hypothenar, forearms, biceps, triceps deltoid and quadriceps femoris, triceps surae was also noted. The gout history and physical examination revealing tophi in multiple joints informed the physician to perform some biochemical tests relating to gout, which found hyperuricemia with a level of 542 umoles/L (normal range, 208428 umoles/L), and the C-reactive protein was $125 \mathrm{mg} / \mathrm{L}$ (normal range, $0-8 \mathrm{mg} / \mathrm{L}$ ).

Based on the symptoms and physical examination, physician intended to consider peripheral neuropathy and could not rule out cervical spondylotic myelopathy. Then the cervical computed tomography (CT) and contrast enhanced magnetic resonance imaging (MRI) were performed. CT scan showed the slightly irregular osteolytic destruction of the C5-6 endplates with discontinuous peripheral sclerotic margins and multiple small calcification and sequestra. Narrowing of C5-6 disc space and mild spinal stenosis were also found (Fig. 1). MR images displayed diffused abnormal signal of the C5 and C6 vertebrae marrow, which were low signal intensity on T1-weighted images and intermediate to high signal intensity on $\mathrm{T} 2$ and short time inversion recovery (STIR) images. The erosion and destruction of both adjacent C5-6 endplates showed discontinuous and waved changes. The C5-6 intervertebral disc appeared fluid like signal changes that were hypointense on $\mathrm{T} 1$ and hyperintense on T2-weighted images. Swelling of prevertebral soft tissue was also found. Contrast-enhanced images showed significant enhancement of the C5-6 vertebrae marrow with no enhancing intervertebral disc. Spinal dural thickening and enhancement of the C5-6 level could be also seen on the enhanced T1-weighted images (Fig. 2).

\section{Timeline}

The timeline for the development of this case is summarized in Fig. 3.

\section{Diagnostic assessment}

In this case, with clinical features, laboratory tests and imaging studies, physicians initially had difficulty in determining the lesions of C5-6; both infectious spondylodiscitis and spinal gout could not be ruled out. In terms of laboratory tests, it was difficult to judge that hyperuricemia was caused by peripheral gout or new lesions, and that the elevation of $\mathrm{C}$-reactive protein was caused by gout or infection. As for the imaging studies of low specificity, radiologist was not completely sure of the etiology and such case was rarely met at that time; initially the imaging reported the first consideration was infectious spondylodiscitis. Under the circumstance of the 


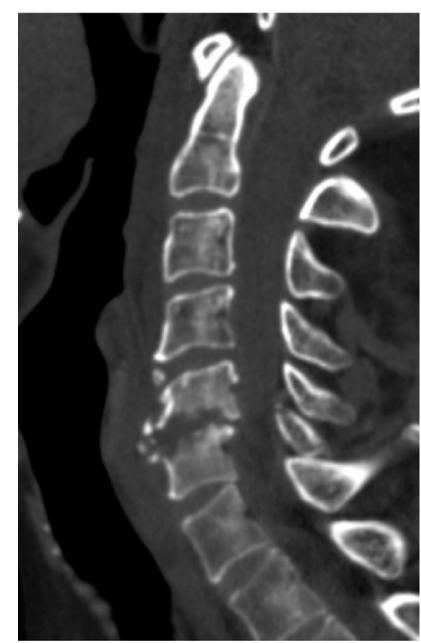

A

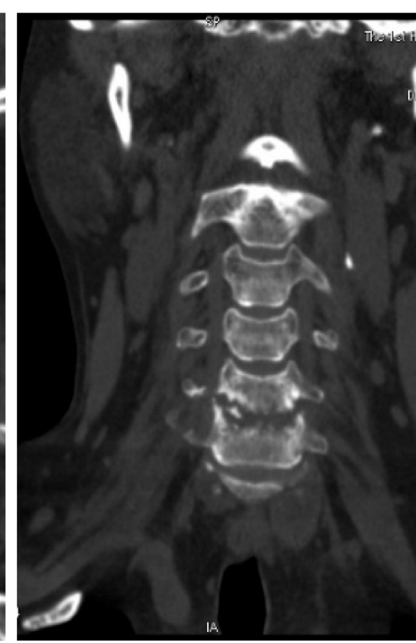

B

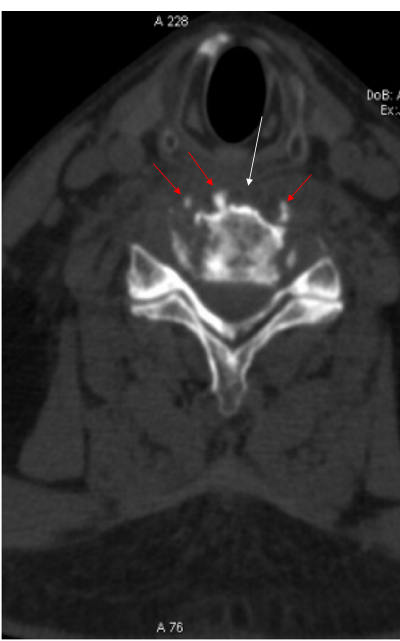

C

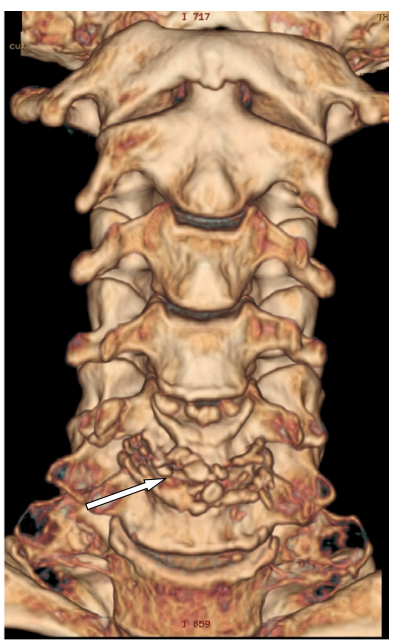

$\mathrm{D}$

Fig. 1 CT image of gout involved the cervical disc and adjacent vertebral endplates in a 50-year-old man with progressive muscular atrophy and weakness in both arms for 9 months and numbness of limbs for 2 months. a Sagittal reconstruction of the cervical spine CT shows slightly irregular osteolytic destruction of C5-6 endplates with discontinuous peripheral sclerotic margins and multiple sequestra and calcification, as well as the narrowing of C5-6 disc space. b Coronal reconstruction CT image illustrating the same findings. $\mathbf{c}$ Axial $C T$ image shows irregular marginal destruction of the vertebral body (white arrow) with multiple small sequestra and calcification (red arrows). d Volume rendered (VR) image shows multiple sequestra (white arrow) and the narrowing of disc space

lesions being not determined and nerve root symptoms, surgical treatment was performed, for the purpose of timely decompressing the spinal cord and providing conditions for the recovery of its function. Intraoperative findings showed that intervertebral disc of C5-6 was completely destructed and replaced by turbid fluid and the erosion of adjacent endplates and vertebrae. The pathological examination of the specimen demonstrated deposited uric acid crystals surrounded by granulomatous inflammation, as well as fiber and angiogenesis with a large number of chronic inflammatory cells infiltration (Fig. 4). Postoperatively, the lesions of C5-6 were definitely diagnosed as gout.

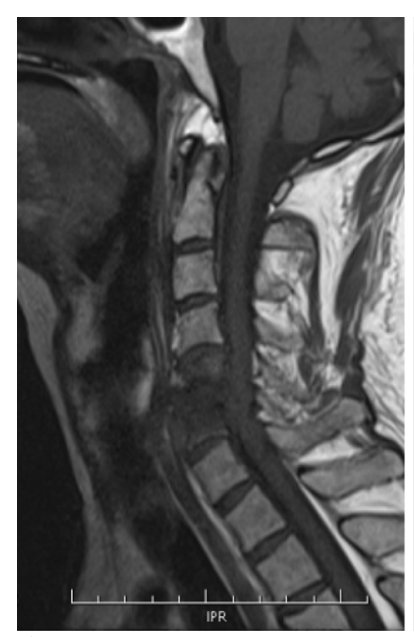

A

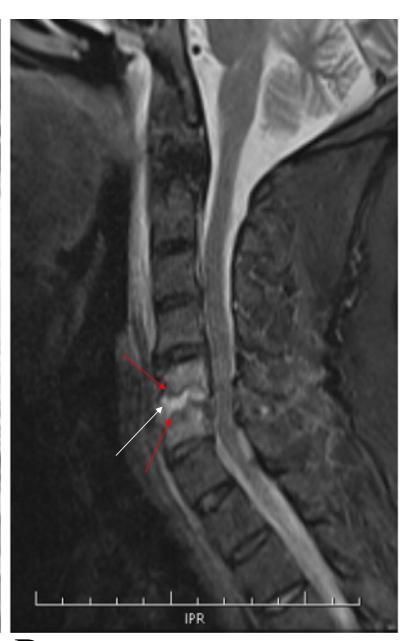

B

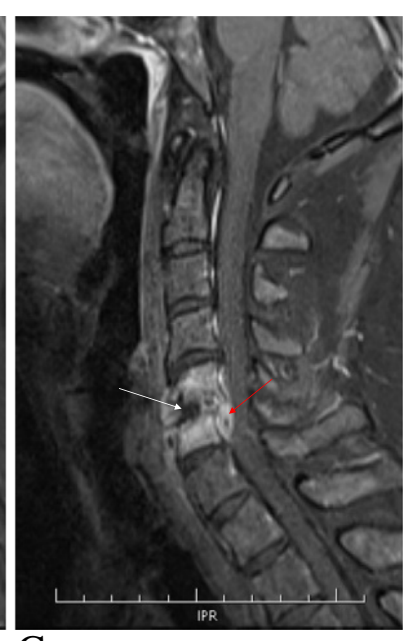

C

Fig. 2 Magnetic resonance (MR) imaging findings in the patient. a Sagittal MR T1-weighted image of the cervical spine showing the normal bone marrow and endplates signal disappeared and replaced by diffused low-signal intensity of the C5 and C6 vertebrae. b Sagittal short time inversion recovery (STIR) MR image illustrating the same diffused high signal intensity of the C5-6 disc (white arrow), the waved erosion and destruction of both adjacent C5-6 endplates (red arrows), and the swelling of prevertebral soft tissue. c Contrast-enhanced sagittal MR T1weighted image with fat saturation illustrating significant enhancement of C5-6 vertebrae marrow with no enhancement of the anterior disc (white arrow), and spinal dural thickening and enhancement (red arrow) 


\section{Timeline for Case Report}

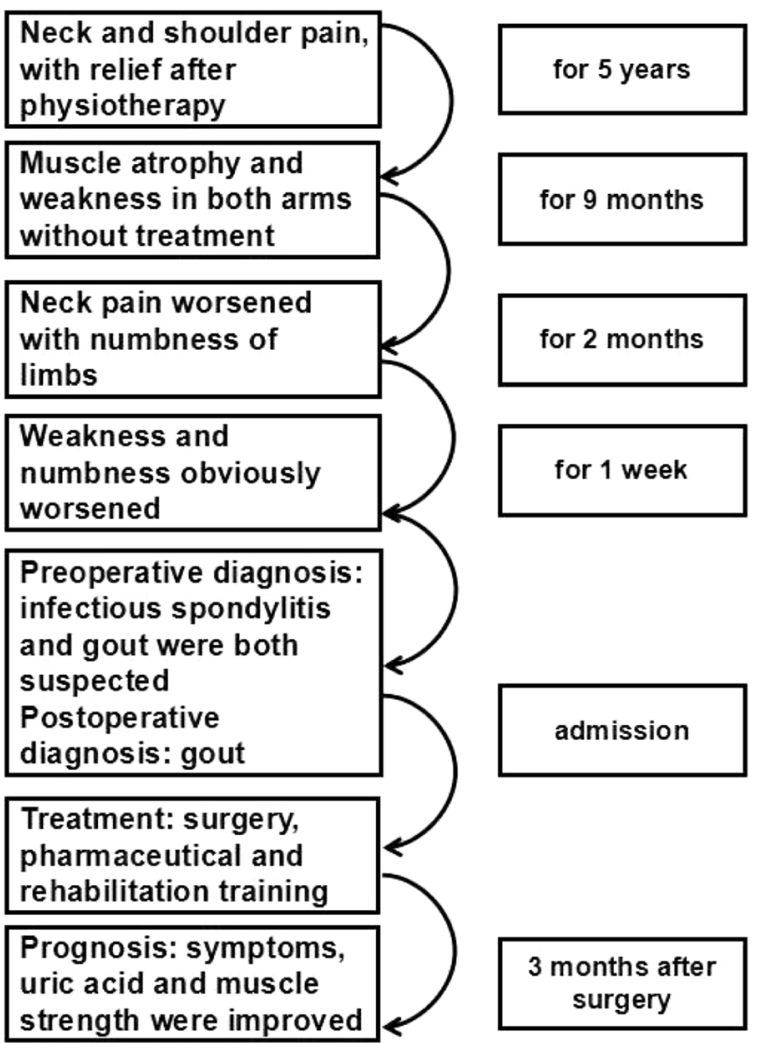

Fig. 3 Timeline of case report. The timeline for the development of this case is summarized in Fig. 3

\section{Therapeutic intervention}

Preoperatively, pharmaceutical treatment was given to relieve pain, nourish nerve and dehydrate [1]. To immediately decompress the nerve root symptoms, surgical treatment including anterior cervical corpectomy, debridement and fusion, and internal fixation of C5-6 was performed $[1,8,11]$. After surgery, the muscle strengths of limbs were significantly improved. Postoperatively, the new diagnosis was confirmed; then colchicine and prednisolone were respectively given $1 \mathrm{mg}$ t.i.d and $10 \mathrm{mg}$ t.i.d. whose dosage reduced when symptoms improved $[1,11]$. Rehabilitation training including electronic biofeedback therapy, pneumatic compression, equipment training and electronic standing bed training was also performed [15].

\section{Follow-up and outcomes}

About 3 months after surgery, the patient felt that pain of neck and shoulder was not obvious and there was no sensory abnormality in the upper limbs; the muscle strengths of limbs were all improved, with upper limbs grade 4 and lower limbs grade 2- to 4 in accordance with Medical Research Council (MRC) Scale for Muscle Strength [14]. The level of serum uric acid was well controlled between 336 umoles/l and 399 umoles/l (normal range, 208-428 umoles/l).

\section{Discussion and conclusions}

In our case, gout-related cervical spine lesions were confirmed and a series of complete and continuous highresolution images including multiplanar reconstruction (MPR), volume rendering (VR) CT images, plain and contrast-enhanced MR images together with exhaustive description were fully displayed. To the best of our knowledge, there have only been 10 such cases with images reported in the English-language literature searched in PubMed (Table 1) [3-11]; most of them just show incomplete and indistinct images. Due to that there were also reports that spinal gout had similar image appearance to infection $[4,5,16,17]$, the main imaging differentiation factors between these conditions should be further discussed.

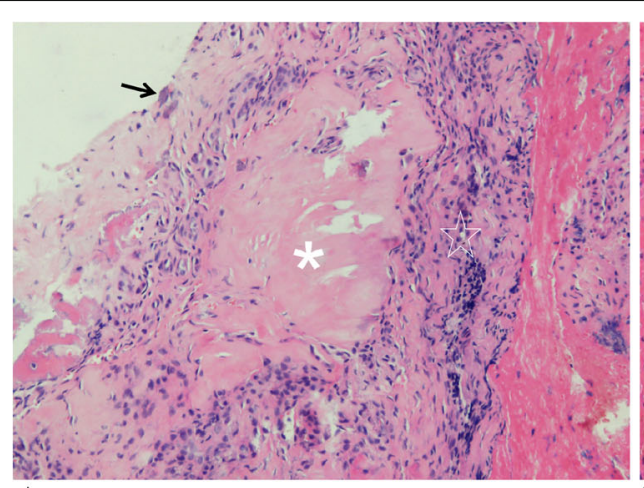

A

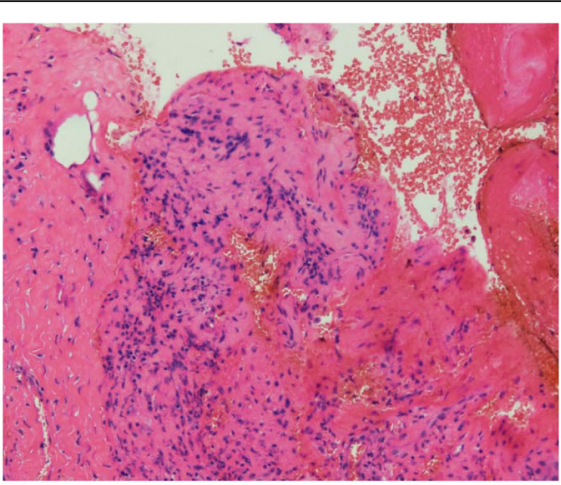

B

Fig. 4 Histological section of a surgically resected specimen of the same patient. Hematoxylin and erosin (HE) staining (a and $\mathbf{b})$ showing the massive deposition of uric acid crystals (a, asterisk) surrounded by granulomatous inflammation (a, pentagon), and few small calcium deposition (a, arrow), as well as fiber and angiogenesis with a large number of chronic inflammatory cells infiltration (b) 


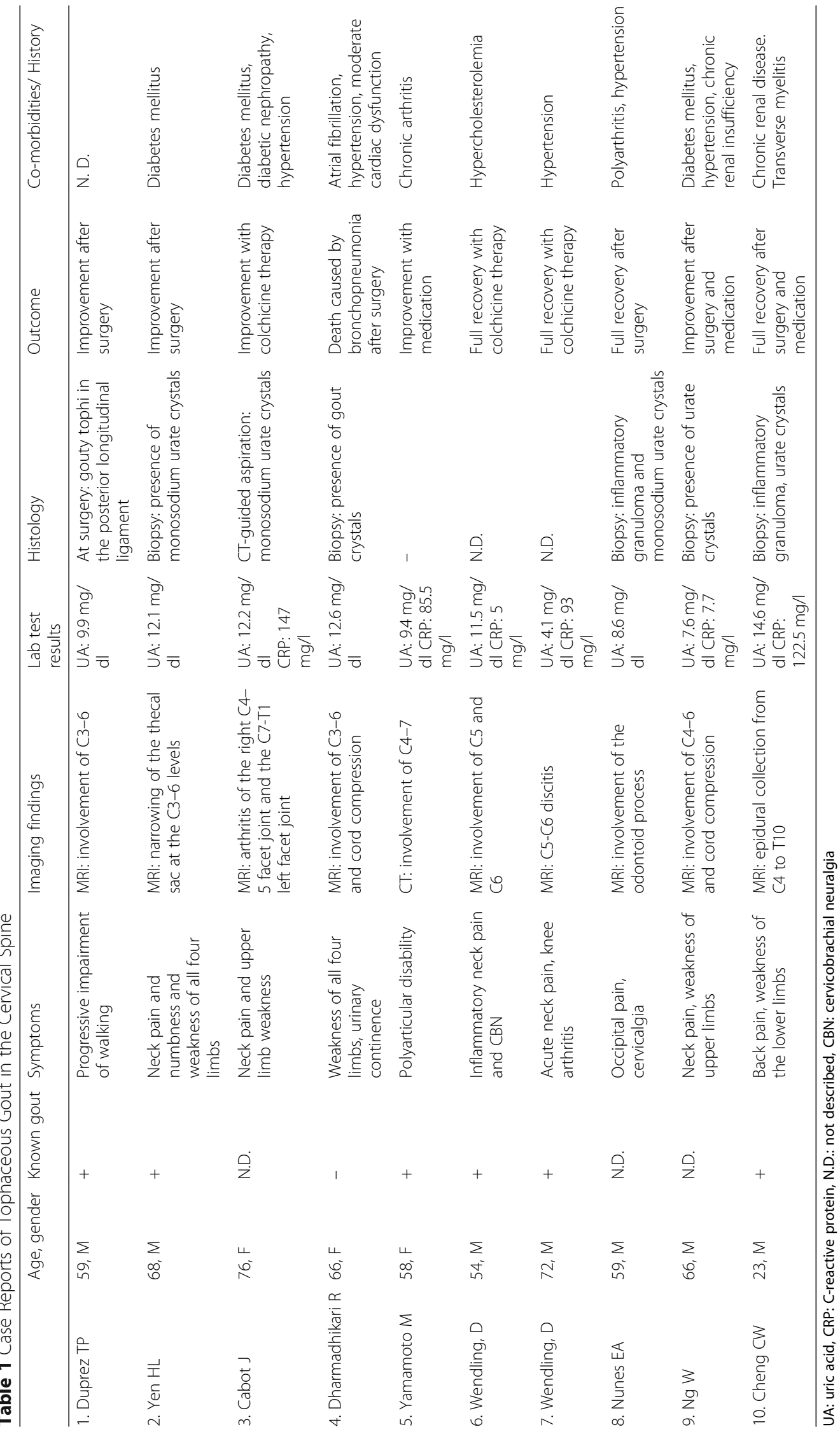


Gouty involvement in intervertebral disc may be rarer than facet joint, which mostly mimics pyogenic discitis $[5,11,16,18]$, but it showed the opposite outcome in the reports reviewed in our article. Considering these 10 reports and including our case, 7 patients' lesions involved discs and only 2 involved facet joints. As for sites of cervical involvement, spinal lesions of 7 patients were seen in the middle cervical segment; 3 of them located in the middle and lower cervical segment, and only $1 \mathrm{pa}-$ tient's lesion located in the upper cervical segment. The lesions can occur in any segment of cervical spine, but are commonly seen in the middle cervical spine C3-6.

The imaging findings of our patient were initially difficult to tell if it was gout or infection. However, looking back on the images, we found that radiological abnormalities for spinal gout may have some difference with common infectious spondylosis according to literature report. Plain films sometimes appear false negative especially in the early stages and subsequent bone changes are advantageous but nonspecific feature of spinal gout $[4,6,7,11]$. CT can help visualize the bone and soft tissue changes caused by tophi which appear as low-density area $[6,7,9,10]$. The typical appearance related to these changes is characterized by punched-out erosion and well-defined margins without surrounding infiltrative changes; structures of vertebrae and endplates can remain unaffected and wedging changes of vertebral bodies are hardly seen [6-10]. MRI appears to be sensitive but nonspecific for the diagnosis of spinal gout, but MRI has an obvious advantage in manifesting abundant information of soft tissue in or around the gouty lesion [19-21]. In the literature review, 5 cases involving the cervical intervertebral discs with MR images was mainly low signal intensity on T1 and T2 weighted images $[4,5,7,9]$. In 2 cases with enhanced MR images, the involved discs were enhanced significantly $[4,9]$. And MR characteristics of 4 patients with tophus were intermediate-to-low signal intensity on T1-weighted images, and on T2-weighted images, the signal intensity varied from low to high $[4,7,9,10]$, which mostly coincided with previous studies $[1,19,20]$. After gadolinium enhancement, the tophi shows homogeneous or heterogeneous enhancement, mainly depending on volumes and distribution of calcification [16]. In the previous studies, the MRI findings have ranged from well-defined punched-out endplate erosive change and narrowing of intervertebral space to severe destructive and proliferative discovertebral changes. And no significant edema was found in the trabecular bone and soft tissue adjacent to the lesions. However, spinal cord edema can sometimes be seen resulting from the cord compression by lesions $[5,7,10,11]$.

Imaging manifestations of spinal gout is not specific and can resemble that of degenerative changes and infectious spondylodiscitis $[4,5,18,22,23]$. Therefore, it is necessary to exclude other probable etiologies. In patients with Modic type 1 vertebral disc changes, reactive sclerosis accompanied by marrow edema usually appears as hypointense on T1-weighted images and hyperintense on T2-weighted and STIR images in acute conditions, and its lesions generally show well defined boundary [24]. For pyogenic spinal infection, disc changes appear as invariably reduced height, non-anatomic T2 hyperintense and enhanced significantly; irregularity, destruction and enhancement of endplates and adjacent vertebral bodies can be seen as well. Inhomogeneous paraspinal inflammatory swelling is common and small abscesses with a limited area can also be formed in some cases [25]. However, vertebral lesions in spinal tuberculosis are usually extensive, causing collapse and malformation in the late stage. Bone destruction, dead bone, narrowing of intervertebral space, angulate deformity and cold abscess are the hallmarks of the disease and can help differentiate it from other conditions, and paraspinal collection occurs more commonly than in pyogenic infection and may be bilateral and disproportionately larger than the degree of bone destruction $[26,27]$. In the cases of spinal brucellosis, the principal features in the sclerotic period are vertebral body hyperplasia, osteophyte proliferation, bony spines resembling "bird beaks" (that sometimes forms bony bridges) and endplate sclerosis; paraspinal soft tissue shadows and predural granulomas are more common than usual, and paravertebral abscesses are less common [27]. The main radiological differentiation factors between these conditions is the characteristics of the osteolytic lesions and changes of paravertebral tissues.

In recent years, a newer method, dual-energy CT (DECT), has been more commonly applied in the differential diagnosis of gout $[3,28,29]$. The sensitivity of DECT in identifying monosodium urate crystals up to $78-100 \%$ makes it more competitive than other methods of imaging. The urate depositions can be clearly seen on DECT, which is able to directly measure the size and volume of depositions [1]. Therefore, DECT apparently has diagnostic potential for patients with an unclear diagnosis or atypical clinical manifestations [22].

Clinically, it is necessary for physicians to break the boundary of the reasoning mechanism that local lesions in the intervertebral discs and adjacent vertebral endplates are just degenerative changes, infectious disease or tuberculosis; henceforth, gout should be higher on the list of differentials especially for the patients not responding to conservative measures. In laboratory tests, hyperuricemia and the elevated level of C-reactive protein were also uncertain that they were caused by gout of limbs or the new lesions, and the new lesions were gout or infection. Blood culture should be supplemented to exclude the possibility of infection when the lesion was difficult to define. Definite diagnosis of gout was 
most commonly made during surgery. In the 10 clinical cases, surgery was performed in 6 cases. An additional 1 case was diagnosed via needle aspiration. In 3 cases, the patients were diagnosed on the basis of blood culture, other clinical data, a history of gout and a rapid response to colchicine. Similar to the majority, etiology of our case was completely confirmed during surgery.

Treatment for spinal gout commonly includes conservative medication and surgery. Owing to the fact that data in most literature suggests that spinal gout may contribute to paraplegia, early intervention is needed after a definite diagnosis. Decompression surgery followed by pharmacological treatment of lowering serum uric acid may be the first treatment for the majority of patients [1]. If necessary, individual prophylaxis and therapeutic strategies can be provided as well.

Cervical spinal gout involving the disc and adjacent vertebral endplates is uncommon and may misunderstand degenerative changes and infectious spondylodiscitis. When such this condition with atypical symptoms and low specific imaging studies is encountered clinically, physician and radiologist should take the gouty spondylitis into account with a combination with previous history and clinical manifestations.

\section{Abbreviations}

CT: Computed tomography; DECT: Dual-energy computed tomography; DM: Diabetes mellitus; MPR: Multiplanar reconstruction; MRI: Magnetic resonance imaging; STIR: Short time inversion recovery; VR: volume rendering

\section{Acknowledgements}

Not applicable.

\section{Authors' contributions}

SZ was involved in manuscript writing. YX was involved in case research. $X L$ was involved in pathological data collection. $Y Z$ was involved in clinical case data collection. HY was involved in study design and management. All authors have read and approved the final version of this manuscript.

\section{Authors' information}

Department of Radiology, the First Affiliated Hospital of Chongqing Medical University, 1 Youyi Road, Yuzhong District, Chongqing 400016, China. Suying Zhou, Yundan Xiao, Xin Liu, Yi Zhong, Haitao Yang (corresponding author).

\section{Funding}

No funding was obtained for this study.

\section{Availability of data and materials}

All data generated or analysed during this study are included in this article and other published articles in the reference.

\section{Ethics approval and consent to participate}

Not applicable.

\section{Consent for publication}

Written informed consent was obtained from the patient for publication of this case report and any accompanying images. A copy of the written consent is available for review by the Editor of this journal.

\section{Competing interests}

The authors declare that they have no competing interests.
Received: 5 July 2019 Accepted: 3 September 2019

Published online: 14 September 2019

\section{References}

1. Toprover M, Krasnokutsky S, Pillinger MH. Gout in the spine: imaging, diagnosis, and outcomes. Curr Rheumatol Rep. 2015;17(12):70.

2. Hausch R, Wilkerson M, Singh E, Reyes C, Harrington T. Tophaceous gout of the thoracic spine presenting as back pain and fever. J Clin Rheumatol. 1999:5(6):335-41.

3. Cheng CW, Nguyen QT, Zhou H. Tophaceous gout of the cervical and thoracic spine with concomitant epidural infection. AME Case Rep. 2018;2:35.

4. Duprez TP, Malghem J, Vande Berg BC, Noel HM, Munting EA, Maldague BE. Gout in the cervical spine: MR pattern mimicking diskovertebral infection. AJNR Am J Neuroradiol. 1996;17(1):151-3.

5. Yen $\mathrm{HL}$, Cheng $\mathrm{CH}$, Lin JW. Cervical myelopathy due to gouty tophi in the intervertebral disc space. Acta Neurochir. 2002;144(2):205-7.

6. Cabot J, Mosel L, Kong A, Hayward M. Tophaceous gout in the cervical spine. Skelet Radiol. 2005;34(12):803-6.

7. Dharmadhikari R, Dildey P, Hide IG. A rare cause of spinal cord compression: imaging appearances of gout of the cervical spine. Skelet Radiol. 2006; 35(12):942-5.

8. Yamamoto M, Tabeya T, Masaki Y, Suzuki C, Naishiro $Y$, Ishigami K, et al. Tophaceous gout in the cervical spine. Intern Med. 2012;51(3):325-8.

9. Wendling D, Prati C, Hoen B, Godard J, Vidon C, Godfrin-Valnet M, et al. When gout involves the spine: five patients including two inaugural cases. Joint Bone Spine. 2013:80(6):656-9.

10. Nunes EA, Rosseti AG Jr, Ribeiro DS, Santiago M. Gout initially mimicking rheumatoid arthritis and later cervical spine involvement. Case Rep Rheumatol. 2014:2014:357826.

11. Ng W, Sin $\mathrm{CH}$, Wong $\mathrm{CH}$, Chiu WF, Chung OM. Unusual presentation of spinal gout: 2 cases report and literature review. J Orthop Case Rep. 2017; 7(6):50-4.

12. Riley D. 2013 CARE Checklist. https://www.care-statement.org/resources/ checklist. Accessed by 4 Jul 2019

13. Malanga G, Nadler S. Musculoskeletal physical examination: an evidencebased approach. Hanley and Belfus; 2006.

14. Medical Research Council. Aids to the examination of the periphera nervous system. In: Memorandum no. 45, her Majesty's stationery office. London; 1981

15. Finn Bjarke C. Lumbar spinal fusion: outcome in relation to surgical methods, choice of implant and postoperative rehabilitation. Acta Orthop Scand Suppl. 2004;75(313):2-43.

16. Yen PS, Lin JF, Chen SY, Lin SZ. Tophaceous gout of the lumbar spine mimicking infectious spondylodiscitis and epidural abscess: MR imaging findings. J Clin Neurosci. 2005;12(1):44-6.

17. Bonaldi VM, Duong H, Starr MR, Sarazin L, Richardson J. Tophaceous gout of the lumbar spine mimicking an epidural abscess: MR features. AJNR Am J Neuroradiol. 1996:17(10):1949-52

18. Suk KS, Kim KT, Lee SH, Park SW, Park YK. Tophaceous gout of the lumbar spine mimicking pyogenic discitis. Spine J. 2007:7(1):94-9.

19. Hsu CY, Shih TT, Huang KM, Chen PQ, Sheu JJ, Li YW. Tophaceous gout of the spine: MR imaging features. Clin Radiol. 2002;57(10):919-25.

20. Tsai $\mathrm{CH}$, Chen YJ, Hsu HC, Chen HT. Bacteremia coexisting with tophaceous gout of the spine mimicking spondylodiscitis: a case report. Spine. 2009; 34(2):E106-9.

21. McQueen FM, Doyle A, Dalbeth N. Imaging in gout--what can we learn from MRI, CT, DECT and US? Arthritis Res Ther. 2011;13(6):246.

22. Willner N, Monoranu CM, Stetter C, Ernestus RI, Westermaier T. Gout tophus on an intradural fascicle: a case description. Eur Spine J. 2016;25(Suppl 1): 162-6.

23. Boody BS, Tarazona DA, Vaccaro AR. Evaluation and Management of Pyogenic and Tubercular Spine Infections. Curr Rev Musculoskelet Med. 2018;11(4):643-52.

24. Kuisma M, Karppinen J, Haapea M, Lammentausta E, Niinimaki J, Tervonen O. Modic changes in vertebral endplates: a comparison of MR imaging and multislice CT. Skelet Radiol. 2009:38(2):141-7.

25. Tali ET, Oner AY, Koc AM. Pyogenic spinal infections. Neuroimaging Clin N Am. 2015:25(2):193-208

26. Kilborn T, Janse van Rensburg P, Candy S. Pediatric and adult spinal tuberculosis imaging and pathophysiology. Neuroimaging Clin N Am. 2015; 25(2):209-31. 
27. Tu L, Liu X, Gu W, Wang Z, Zhang E, Kahar A, et al. Imaging-assisted diagnosis and characteristics of suspected spinal brucellosis: a retrospective study of 72 cases. Med Sci Monit. 2018;24:2647-54.

28. Chou H, Chin TY, Peh WC. Dual-energy CT in gout - a review of current concepts and applications. J Med Radiat Sci. 2017;64(1):41-51.

29. Jegapragasan M, Calniquer A, Hwang WD, Nguyen QT, Child Z. A case of tophaceous gout in the lumbar spine: a review of the literature and treatment recommendations. Evid Based Spine Care J. 2014;5(1):52-6.

\section{Publisher's Note}

Springer Nature remains neutral with regard to jurisdictional claims in published maps and institutional affiliations.

Ready to submit your research? Choose BMC and benefit from:

- fast, convenient online submission

- thorough peer review by experienced researchers in your field

- rapid publication on acceptance

- support for research data, including large and complex data types

- gold Open Access which fosters wider collaboration and increased citations

- maximum visibility for your research: over $100 \mathrm{M}$ website views per year

At $\mathrm{BMC}$, research is always in progress.

Learn more biomedcentral.com/submissions 